\title{
The effect of intranasal oxytocin on visual processing and salience of human faces
}

\author{
Daniel Hovey (1D', Louise Martens $\mathbb{B}^{2,3}$, Bruno Laeng $\mathbb{B}^{4,5}$, Siri Leknes ${ }^{4}$ and Lars Westberg
}

\begin{abstract}
The mechanisms underlying the role of oxytocin (OT) as a regulator of social behavior in mammals are only partly understood. Recently, it has been proposed that OT increases the salience of social stimuli. We carried out a randomized, double-blind, cross-over study of the effects of OT on binocular rivalry, a visual phenomenon underpinned by the interplay of excitation and inhibition in the cortex. A final sample of 45 participants viewed images of social stimuli (faces with different emotional expressions) and non-social stimuli (houses and Gabor patches). We demonstrate a robust effect that intranasal OT increases the salience of human faces in binocular rivalry, such that dominance durations of faces are longer-this effect is not modulated by the facial expression. We tentatively show that OT treatment increases dominance durations for non-social stimuli. Our results lend support to the social salience hypothesis of OT, and in addition offer provisional support for the role of OT in influencing excitation-inhibition balance in the brain.
\end{abstract}

\section{Introduction}

The neuropeptide oxytocin (OT) has long been implicated in the regulation of social behavior in mammals, including humans ${ }^{1}$. The exact role of $\mathrm{OT}$ in this regulation, and the mechanisms behind it, are only partly understood. The picture is complicated by the wealth of studies reporting varying results of the administration of intranasal $\mathrm{OT}$, including both prosocial and antisocial effects $^{2,3}$. Several hypotheses have been proposed to explain the influence of OT on social behavior.

The social salience hypothesis suggests that OT increases the salience of social cues, which in turn can increase or modulate the influence of context and interindividual factors on social behavior ${ }^{4,5}$. While the effects of exogenously administered OT are not confined solely to social behavior or social cognition, many studies have indicated that exogenously administered OT affects for

\footnotetext{
Correspondence: Daniel Hovey (daniel.hovey@neuro.gu.se)

${ }^{1}$ Department of Pharmacology, Institute of Neuroscience and Physiology, The

Sahlgrenska Academy, University of Gothenburg, Gothenburg, Sweden

${ }^{2}$ Department of Psychiatry and Psychotherapy, University of Tübingen,

Tübingen, Germany

Full list of author information is available at the end of the article

Open access funding provided by University of Gothenburg.
}

example, memory for faces ${ }^{6}$, detection of social words ${ }^{7}$, and learning from social feedback ${ }^{8}$. Such effects have been observed for both positive and negative stimuli ${ }^{9-13}$. While individual studies have reported selective effects on stimuli with either positive or negative valence, taken together evidence is in line with a general increase of the salience of social stimuli, regardless of the valence of those stimuli. Thus, while OT presumably has several different effects on social cognition, the social salience hypothesis would explain much of the results of studies on exogenously administered OT.

Binocular rivalry is a visual phenomenon that occurs when dissimilar images are presented to different eyes (e.g., a face to one eye and a house to the other). The images then compete for visual awareness, i.e. the image that the viewer consciously perceives (dominant percept) is one of the presented images, while the other one is suppressed and unseen. After a few seconds, the suppressed image become dominant and the previously dominant image becomes suppressed. This results in transitions between which image is consciously perceived, and these transitions may be instant or contain a mix of the two images (piecemeal percept). This phenomenon 
has been extensively used to study conscious visual awareness, where a potentially salient visual stimulus can be dominant or suppressed for relatively long periods of time (several seconds) ${ }^{14}$. Thus, studies using binocular rivalry may yield insights regarding information processing and awareness-what enters our conscious minds, and what influences this salience? Previous studies have investigated binocular rivalry in relation to altered social cognition in psychiatric disorders, and shown reduced salience (shorter dominance durations) for positive social cues in the form of smiling faces in social anxiety dis$\operatorname{order}^{15}$, increased initial salience (initial dominant percept) for fearful faces in anxiety disorders ${ }^{16}$, and altered binocular rivalry alternation rates for emotional faces with increased depressive symptoms ${ }^{17,18}$. Simple characteristics of the stimuli, such as contrast, spatial frequency, and color, can also influence their strength ${ }^{19,20}$. In addition, the strength of a stimulus can be influenced by what that stimulus is associated with, such as neutral faces paired with negative gossip being more dominant than those paired with positive or neutral gossip ${ }^{21}$-demonstrating clear top-down influences on visual consciousness.

Classical accounts of the neural underpinnings of binocular rivalry proposed a reciprocal and fluctuating lateral inhibition of the visual cortices ${ }^{22}$. Later models incorporated inhibitory and excitatory components, as well as topdown influences ${ }^{14,23,24}$, such as attentional aspects, and meaningfulness of the stimulus. While the specific mechanics of this top-down influence remain to be determined, the reciprocal action in the primary visual cortices is believed to depend on the balance of excitation ${ }^{23,25}$ and inhibition $^{26,27}$. A link between binocular rivalry and excitation-inhibition balance is also supported by findings in individuals with autism spectrum disorder (ASD) $)^{28-30}$, where that balance is thought to be disturbed ${ }^{31,32}$.

Accordingly, binocular rivalry offers a so far untapped opportunity to investigate how OT may influence the salience of social cues in humans. Using for the first time binocular rivalry in combination with intranasal OT treatment, and in line with the social salience hypothesis, we hypothesized that the dominance of a face (i.e., a visual stimulus rich in social cues) would increase when exogenous OT is administered, and that this effect should be specific to social stimuli, as well as independent of the affective valence of the face.

\section{Methods}

\section{Participants}

A total of 50 healthy male volunteers (mean age $=$ $27.9 \pm 6.2$ years) were recruited through advertising online and at the local campus, and were monetarily compensated with $300 \mathrm{NOK}$ (approximately 34 USD). All participants had normal visual acuity, either unaided or with correction, and reported no eye conditions (such as amblyopia, strabismus, or diplopia) or any pertinent medical conditions. Color blindness was screened for using Ishihara color plates. Following a description of the aim of the study and the procedures involved, written consent was obtained. The study was approved by the Regional Ethics Committee (2009/208/REK sør-øst C).

\section{Drug protocol}

OT (Syntocinon ${ }^{\circledR}$, Novartis) and placebo (normal saline, $0.9 \%$, Miwana) were administered in the form of nasal spray in identical conventional pump-actuated bottles (five puffs in each nostril). OT was administered in a dose of $40 \mathrm{IUs}$, as in our previous study ${ }^{33}$. The study used a randomized, double-blind, placebo-controlled, cross-over design, such that all subjects received both OT and placebo treatment over the course of two visits on separate days. Using Latin squares, participants were randomly assigned to receive either OT or placebo on their first visit. The participants as well as the experimenter were blinded as to whether they received OT or placebo on their separate visits. The average length of time between visits was 5.73 days (range $3-12$ days).

Participants were verbally instructed to self-administer the intranasal spray in each nostril, and were explicitly instructed to sniff well and to not tilt their head back during the administration. At the end of each visit, the participants were asked whether or not they thought they received OT, and did not perform better than chance $\left(57 \%\right.$ correct; $\left.\chi^{2}(1)=1.089 ; p>0.05\right)$. Since the test described below was part of a battery of four brief tests, participants were randomized for the order of these tests, and this order was then kept constant between sessions. The test described below would thus take place 30-60 min after administration of OT or placebo.

\section{Stimuli}

During each of the two visits, participants viewed a series of 30 stationary anaglyphs-please see Fig. 1 for examples $^{34}$. Of these, 28 consisted of an image of a female face (sourced from the Karolinska Directed Emotional Faces) and a superimposed house image (public domain image available online), where the face would be either red or cyan, and the house would have the opposite color. Half of the anaglyphs had a red face and half had a cyan face. The face displayed one of six emotional expressions - joy, anger, sadness, surprise, fear, or disgust-or had a neutral expression. Thus, four anaglyphs out of the 28 would display each expression. Two final anaglyphs consisted of Gabor patches, i.e., intersecting vertical and horizontal low spatial frequency lines. Gabor patches are thus visual stimuli with low complexity and no social information. One of the pictures had red vertical lines and cyan horizontal lines, and one had the opposite arrangement. See Fig. 1 for examples of face/house and Gabor 
a

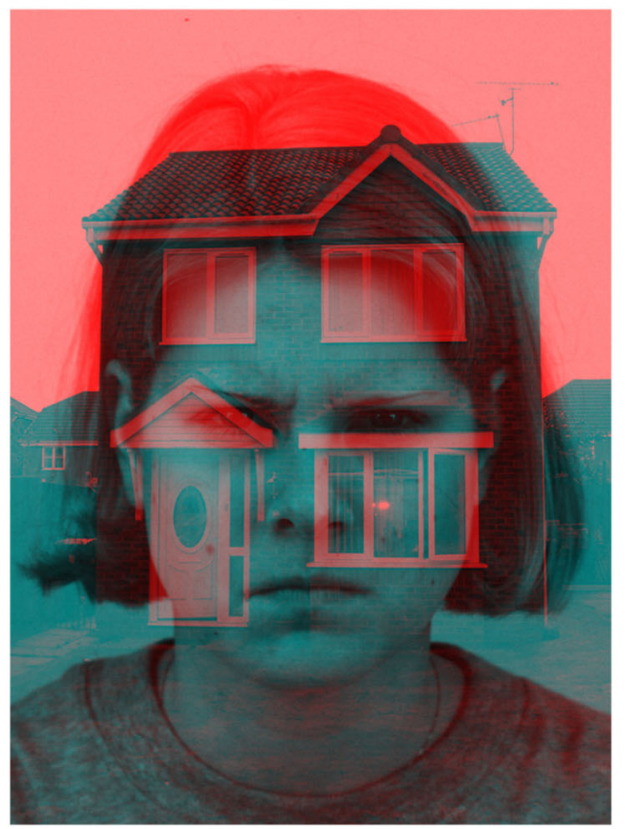

C b

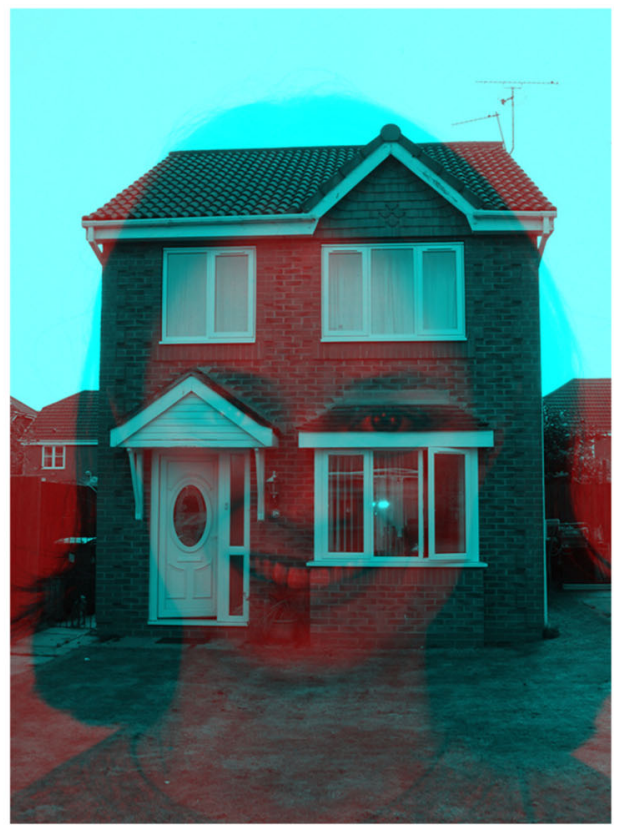

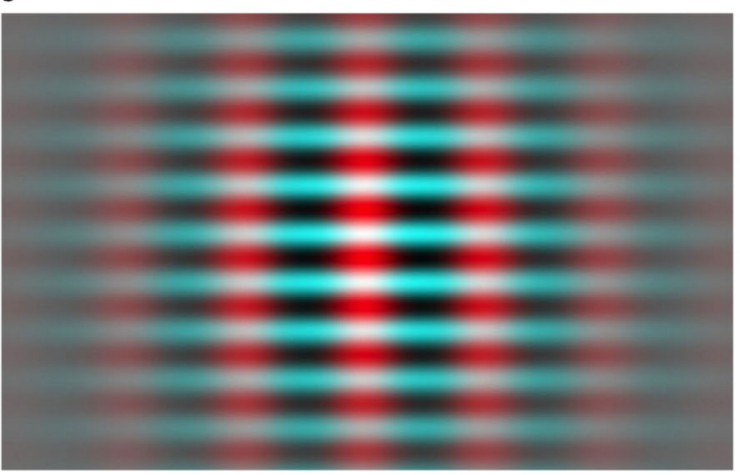

Fig. 1 Anaglyph examples. a Face/house anaglyph. b Face/house anaglyph with inverted colors. c Gabor anaglyph.

anaglyphs. The anaglyphs were presented using SMI Experiment Center (SensoMotoric Instruments, RED500, $\mathrm{SMI}^{\odot}$ Berlin, Germany) on a stationary computer. The anaglyphs were presented at an approximate viewing angle of 38 degrees, with participants positioned at a distance of $70 \mathrm{~cm}$ from the computer screen.

\section{Experimental procedure}

Each participant was provided with colored anaglyph glasses (red and cyan), and received instructions prior to a brief practice trial. Each experimental run consisted of the 28 face/house anaglyphs being presented in sequence to the participant. Each anaglyph would be presented for $30 \mathrm{~s}$, with one anaglyph directly following the next. This sequence of 28 anaglyphs were then followed by a brief one minute break, after which the participant then viewed the two Gabor anaglyphs, which would be presented for
$60 \mathrm{~s}$ each. The participant self-reported dominant or piecemeal percepts by pressing one of three buttons to indicate what they perceived. Button presses were registered with a single time stamp, regardless of how long the button was pressed. While viewing the face/house anaglyphs, the left button would indicate face dominance, the right one house dominance, and the middle one piecemeal percept. During viewing of the Gabor anaglyphs, the left and right buttons would indicate dominant red or cyan percept, respectively, and the middle one piecemeal percept.

\section{Data analysis and statistics}

Data retrieved from BeGaze software $\left(\mathrm{SMI}^{\odot}\right.$ Berlin, Germany) contained timed key presses for each of the separate anaglyphs. Data were extracted using an in-house script written in Python 3.4 (see Supplementary Material). 
We extracted durations of dominance (ms) of uninterrupted percepts for all anaglyphs, categorized as face, house, and Gabor dominance durations, respectively. The time before the initial button press for each anaglyph was excluded, since there was no way to accurately determine whether the indicated percept before initial key press was in fact what the participant was seeing, or if it was caused by bleed-over from the previous anaglyph, or simply reaction time latency. Similarly, the time following the ultimate button press for each anaglyph was also excluded, to avoid artificially shortened durations. All dominance duration means were calculated following an initial exclusion of dominance durations shorter than 250 milliseconds or longer than the mean plus three standard deviations across all participants for the two types of anaglyph (face/house and Gabor, respectively). We extracted average durations of face dominance duration for all separate emotions (seven variables per session), and also averaged durations across all emotions for an overall mean duration of face dominance duration (one variable per session). House dominance durations were averaged across all emotions (one variable per session). Gabor dominance was averaged across both dominance percepts (one variable per session).

We also calculated alternation rates for face/house anaglyphs and Gabor anaglyphs, respectively, since this has been previously used to investigate excitation-inhibition balance $^{30}$. An alternation was defined as a complete transition from one dominant percept to another, and alternation rate was quantified as the number of alternations per $30 \mathrm{~s}$.

Five participants were excluded from statistical analyses for consistently displaying no meaningful rivalry (i.e., less than two key presses for more than $50 \%$ of stimuli in either or both of the sessions) or misunderstanding the instructions. This yielded a final sample of 45 participants. In order to ensure good quality data, results from individual stimuli were excluded if they contained less than two key presses, leading to a data loss of $3.9 \%$ from the $n=45$ sample.

The data were analyzed using SPSS 23 . We utilized repeated measures analysis of variance (RM-ANOVA) to analyze average dominance durations between different dominant percepts (face, house, and Gabor), where percept type and drug were within-subject factors. RMANOVA was also used to investigate the modulation of dominance durations for face percepts and face predominance by emotional content, where drug and emotion were within-subject factors. Post-hoc tests were paired t-tests. Alternation rates were tested using paired $t$-tests for the average rates across face/house and Gabor anaglyphs, respectively. All tests were two-tailed.

We did not conduct a specific power analysis prior to data collection for the binocular rivalry task. With 45 people per group in a between-subjects study, for a twotailed contrast ( $t$-test) $G^{*}$ power estimates $80 \%$ power to detect a medium effect, $d=0.6$. However, in withinsubjects studies of drug effects on behavioral task outcomes, correlations between drug and placebo are typically 0.6 or higher ${ }^{35-39}$. In our data, the correlation coefficient of average dominance durations of faces, houses, and gabors between drug conditions ranged from $r=0.687$ to $r=0.829$. Assuming $r=0.6$ between oxytocin and placebo condition measures of binocular rivalry based on previous literature only, our within-subjects study is at least as sensitive as a between-subjects study with 75 participants per group, i.e. has $80 \%$ power to detect $d=$ 0.46 . This means that even considering the boost in statistical power yielded by our design, the study lacked the sensitivity to detect small effects.

\section{Results}

Please see Table 1 for all pairwise comparisons mentioned below.

\section{Dominance durations}

Average dominance durations (ms) of uninterrupted percepts were calculated for face (across all emotions), house, and Gabor percepts. Using RM-ANOVA with a 2 (oxytocin/placebo) $\times 3$ (face, house, or Gabor percept) design, we found a significant main effect of drug $\left(F(1,42)=10.64 ; p=0.002 ; \eta p^{2}=0.202\right)$ and of percept $\left(F(2,41)=45.74 ; p<0.001 ; \eta p^{2}=0.691\right)$, as well as an interaction between drug and percept $(F(2,41)=3.50$; $p=0.039 ; \eta p^{2}=0.146$ ). Subsequent post-hoc analyses revealed that compared to the placebo treatment, OT significantly increased face dominance durations for all emotions combined $\left(t(44)=3.86 ; p<0.001 ; \eta p^{2}=0.253\right.$; Fig. 2a).

The similar combined measure for house dominance durations was on the border of statistical significance for OT treatment increasing dominance durations for houses as well $\left(t(44)=1.69 ; p=0.098 ; \eta p^{2}=0.061\right.$; Fig. $\left.2 \mathrm{~b}\right)$, compared to placebo. Similarly, Gabor dominance durations showed a trend approaching significance for being increased in the OT condition compared to the placebo condition $\left(t(42)=1.86 ; p=0.069 ; \eta p^{2}=0.076\right.$; Fig. 2c).

Dominance durations for faces were also averaged separately for the different emotions. RM-ANOVA analysis with a 2 (oxytocin/placebo) $\times 7$ (emotions) was performed, with Mauchly's test of sphericity indicating that sphericity was violated for drug $\times$ emotion $(W=0.221$; $p<0.001)$. The degrees of freedom were thus corrected using Huyhn-Feldt estimates of sphericity. There was no significant interaction between drug and emotion of the face perceived on dominance durations $(F(5,200)=0.975$; $p=0.431)$. The face dominance durations were higher with oxytocin for all emotional face categories. Post-hoc 
Table 1 Pairwise comparisons for percepts/anaglyphs.

\begin{tabular}{|c|c|c|c|c|c|c|}
\hline Variable & Percept/Anaglyph & Placebo & Oxytocin & df & $t$ & $p$ \\
\hline \multirow[t]{10}{*}{ Average dominance duration (ms) } & Face-Combined & $2363 \pm 125$ & $2707 \pm 158$ & 44 & 3.86 & 0.0004 \\
\hline & Face-Joy & $2399 \pm 163$ & $2650 \pm 181$ & 43 & 1.31 & 0.2 \\
\hline & Face-Anger & $2358 \pm 143$ & $2768 \pm 216$ & 43 & 1.96 & 0.057 \\
\hline & Face_-Sadness & $2327 \pm 142$ & $2785 \pm 190$ & 43 & 3.41 & 0.001 \\
\hline & Face-Surprise & $2431 \pm 139$ & $2674 \pm 165$ & 43 & 1.68 & 0.1 \\
\hline & Face-Fear & $2340 \pm 171$ & $2728 \pm 219$ & 44 & 1.87 & 0.068 \\
\hline & Face—Disgust & $2152 \pm 125$ & $2719 \pm 205$ & 44 & 3.2 & 0.003 \\
\hline & Face-Neutral & $2561 \pm 193$ & $2618 \pm 169$ & 42 & 0.29 & 0.78 \\
\hline & House & $2639 \pm 105$ & $2806 \pm 134$ & 44 & 1.69 & 0.098 \\
\hline & Gabor & $1658 \pm 66$ & $1775 \pm 91$ & 42 & 1.86 & 0.069 \\
\hline \multirow[t]{2}{*}{ Average piecemeal duration (ms) } & Face/House & $2601 \pm 124$ & $2677 \pm 184$ & 44 & 0.41 & 0.68 \\
\hline & Gabor & $1523 \pm 113$ & $1583 \pm 126$ & 41 & 0.42 & 0.68 \\
\hline Average alternation rate & Face/House & $3.6 \pm 0.4$ & $3.9 \pm 0.3$ & 44 & 1.26 & 0.22 \\
\hline (switches/30s) & Gabor & $10.5 \pm 0.6$ & $9.6 \pm 0.6$ & 42 & 1.45 & 0.16 \\
\hline
\end{tabular}

All means presented with standard error of the mean.

paired $t$-tests comparing oxytocin and placebo reached significance for sadness and disgust. The mean difference in dominance durations was least apparent for the neutral condition (Fig. 2d).

There were no statistically significant differences in piecemeal durations, for face/house stimuli or for Gabor stimuli (all $p>0.05$ ).

\section{Alternation rates}

We included alternation rates as an outcome since this has been previously utilized in order to investigate excitation-inhibition balance with binocular rivalry ${ }^{30}$. There was, however, no significant difference between intranasal oxytocin and placebo with regards to alternation rate between the face and house percept, defined as alternations $/ 30 \mathrm{~s}(t(44)=1.18 ; p=0.25)$. There was also no significant difference between the oxytocin and placebo condition with regards to alternation rate between dominant Gabor percepts, defined as switches $/ 30 \mathrm{~s}$ $(t(42)=1.45 ; p=0.16)$.

\section{Discussion}

To our knowledge, this is the first study to utilize binocular rivalry to investigate the effects of intranasal OT on visual processing. Longer dominance durations for faces during binocular rivalry confirmed our hypothesis that intranasal OT increases the salience of faces. The higher effect size for faces rather than nonsocial stimuli is congruent with the social salience hypothesis ${ }^{4}$; we however, did not find that emotional content significantly modulated the response to OT administration. Our data thus provides support for the idea that OT increases the salience of social signals of positive and negative affective valence. Nevertheless, we also found non-significant but intriguing trends that intranasal OT increases the dominance durations for both Gabor and house percepts-both non-social in nature. We did not find any significant differences in alternation rates between placebo and oxytocin for the face/house anaglyphs, nor for the Gabor anaglyphs.

The increase in salience across valences is in line with studies where the outcome measure can be assumed to depend on the salience of stimuli, for instance showing that intranasal OT increases memory retention of faces $^{6,12}$ and emotion recognition accuracy ${ }^{11,40}$. The role of OT in face and emotion recognition has been stressed by the above mentioned pharmacological as well as genetic studies ${ }^{41-44}$; however, other studies report incongruent results ${ }^{39}$. We would argue that our results are novel in their methodological approach, and lend support to the social salience hypothesis, through a psychophysiological measurement.

There are several potential mechanisms by which OT may influence rivalry dynamics and the processing of visual social information. Firstly, OT may have increased face dominance during binocular rivalry via attentional mechanisms ${ }^{45-48}$. Recent neuroimaging studies using intranasal OT are in line with enhanced visual attention, e.g., enhanced early visual activity for faces ${ }^{49}$, and increased functional connectivity in the nucleus basalis of Meynert, which regulates selective attention ${ }^{50}$. This interpretation of our results is in line with the social 

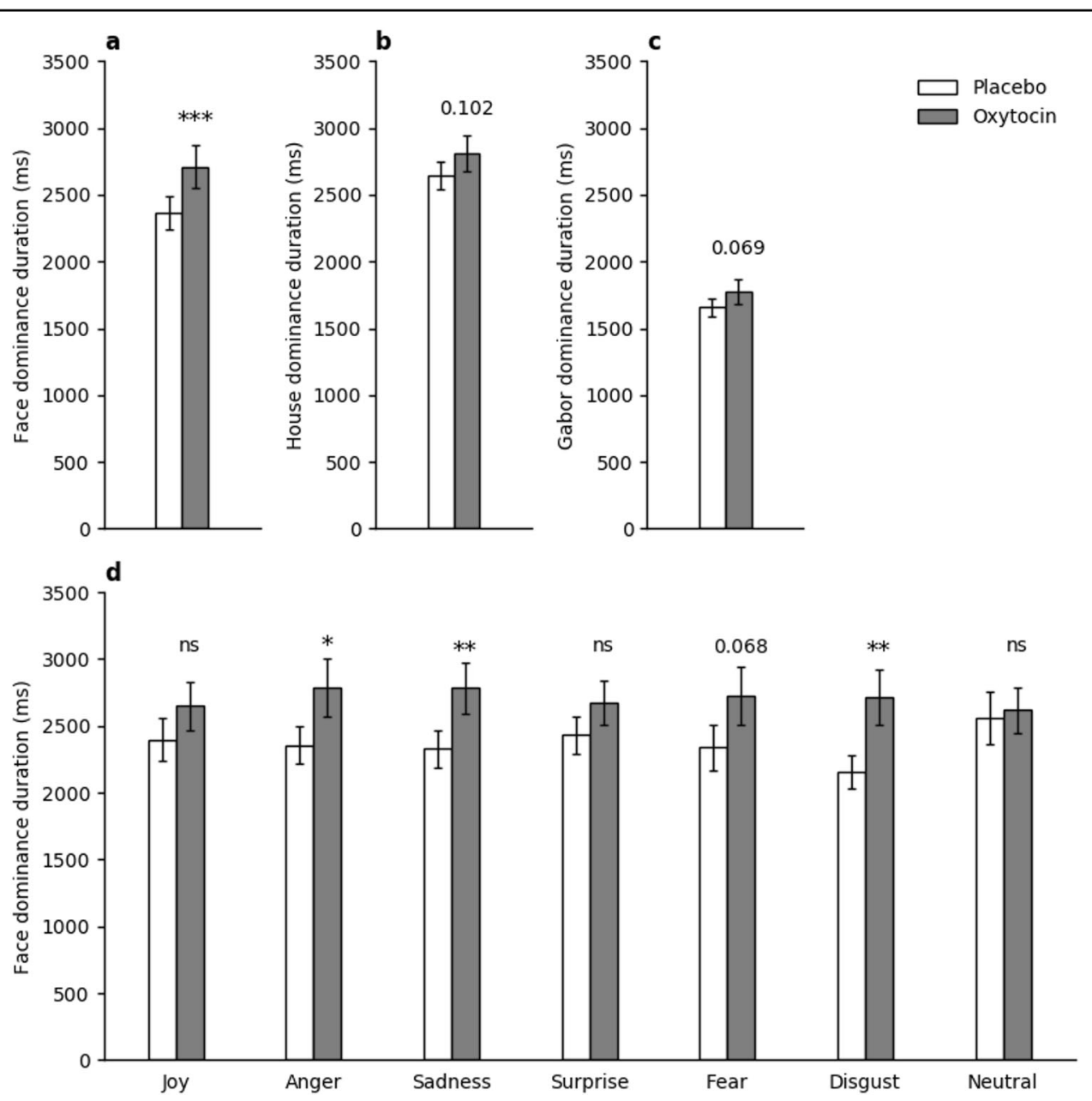

Fig. 2 Dominance duration results. a-c Dominance durations for faces, houses, and Gabor patches, respectively. $\mathbf{d}$ Dominance durations for faces by emotion. Means \pm standard error of the mean. PLC: placebo; OXT: oxytocin.

salience hypothesis, where attention, as guided by oxytocinergic influences on dopaminergic circuitry, is central ${ }^{4}$. Secondly, previous pharmacological studies on the effects of the serotonin receptor agonists psilocybin and tandospirone demonstrated increased dominance durations for Gabor patches ${ }^{51,52}$, suggesting that serotonin acts to modulate binocular rivalry. OT has been shown to directly influence the release of serotonin ${ }^{53}$, in for instance the nucleus accumbens ${ }^{54}$. Serotonergic signaling, therefore, constitutes a potential mediator of OT's effects on rivalry dynamics. Thirdly, binocular rivalry is a phenomenon based on interplay between inhibitory and excitatory neurons, and there is ample evidence that OT is able to influence excitation-inhibition balance in various parts of the brain. For example, it has been implicated in the initial transient switch in $\gamma$-aminobutyric acid (GABA) receptors from depolarizing to hyperpolarizing ${ }^{55-57}$. OT receptor knockout mice display a decreased ratio of GABA-ergic to total presynapses in the hippocampus, and increased seizure susceptibility ${ }^{58}$, and OT has been shown to influence glutamate neurotransmission in brain slices of mice $^{59}$. Intriguingly, in the sensory cortices, OT rapidly alters the excitation-inhibition balance in the auditory cortex to enable maternal behavior in female mice ${ }^{60}$, as well as enhance pre- and postsynaptic glutamatergic signaling in the rat olfactory bulb ${ }^{61}$. While we cannot, based on these three lines of reasoning, pinpoint from our data where OT acts to achieve its effects on rivalry dynamics, our results provide the support that OT does influence the excitation-inhibition balance phenomenon of binocular rivalry, by top-down effects and/or directly in the visual cortex. In addition, these proposed mechanisms may in part explain the fact that we see strong trends towards increased dominance durations for non-social stimuli as well.

Our tentative findings on non-social stimuli are congruent with previous literature indicating that the effects of OT are not restricted to only social contexts, as it may 
mediate for instance approach and avoidance behavior in non-social contexts as well ${ }^{62}$. The approach-avoidance hypothesis of OT has not been specifically addressed here, and our non-social stimuli were arguably simplistic. This makes it difficult to draw any firm conclusions on how our findings may relate to this hypothesis, but provides an intriguing avenue of further research.

OT administration in mammals has been shown to rescue social behavior deficits ${ }^{63}$. Hence, it is not surprising that OT has been suggested as a possible treatment for social impairments in several psychiatric and neurological diagnoses, such as developmental prosopagnosia $^{64}$, borderline personality disorder ${ }^{65}$, and $\mathrm{ASD}^{66}$. This therapeutic option is appealing, given the relatively mild side effect profile of OT, and the ease of intranasal administration; however, the effect duration is relatively short. Substantial controversy remains about how and how much intranasally administered oxytocin reaches the brain in humans ${ }^{67}$, and the few human studies applying variable doses have yet to produce conclusive results ${ }^{68-70}$. The dose applied here (40IU) is the largest dose typically applied in human research. Studies attempting treatment with intranasal OT for individuals with ASD have had encouraging, although somewhat mixed, results ${ }^{63}$. Our findings encourage future studies on exogenously administered OT as a treatment for social impairments, for example, in combination with behavioral therapy to fully utilize the effect duration.

Limitations to the study include that it was not powered to detect small effects, although many intranasal oxytocin studies report small effects. Another potential weakness of our study is that it included only men. Given the well-known sex-specific idiosyncrasies of the OT system $^{71}$, we would urge future studies to include women as well. Furthermore, this study included only female faces, and future studies would do well to investigate if early visual processing of same-sex and oppositesex faces is different. In addition, this study did not include any measure of whether each emotional expression was recognized, which would have allowed a deeper analysis of the possible correlation between a change in early visual processing and aspects of social cognition. Lastly, the participants were not asked whether they had any conditions that may have obstructed the nasal cavity (e.g., past nasal surgery, current cold/flu), which could possibly influence the response to intranasal OT.

In conclusion, we have demonstrated that intranasal OT administration leads to altered visual processing of social stimuli, by increasing dominance durations for faces in a binocular rivalry paradigm. This is an easily administered and non-invasive test, which can be further modified to probe aspects of visual awareness, with additional pharmacological manipulations, which will allow more detailed investigations of the effects of $\mathrm{OT}$ on perception and processing of social stimuli. Our results lend support to the hypothesis that OT increases the salience of social stimuli, and suggests that this increased salience happens across stimuli of different valences.

\section{Acknowledgements}

The authors would like to thank Dr. Nouchine Hadjikhani and Dr. Soaleha Shams for help with critiquing and editing the paper. This research was supported by funding from the Swedish Research Council, an ALF-grant from Region Västra Götaland, and the Wilhelm and Martina Lundgren Foundation.

\section{Author details \\ ${ }^{1}$ Department of Pharmacology, Institute of Neuroscience and Physiology, The Sahlgrenska Academy, University of Gothenburg, Gothenburg, Sweden. ${ }^{2}$ Department of Psychiatry and Psychotherapy, University of Tübingen, Tübingen, Germany. ${ }^{3}$ Max Planck Institute for Biological Cybernetics, Tübingen, Germany. ${ }^{4}$ Department of Psychology, University of Oslo, Oslo, Norway. ${ }^{5}$ RITMO Centre for Interdisciplinary Studies in Rhythm, Time and Motion, Oslo, Norway}

\section{Conflict of interest}

The authors declare that they have no conflict of interest.

\section{Publisher's note}

Springer Nature remains neutral with regard to jurisdictional claims in published maps and institutional affiliations.

Supplementary Information accompanies this paper at (https://doi.org/ 10.1038/s41398-020-00991-3).

Received: 10 August 2018 Revised: 22 June 2020 Accepted: 24 June 2020 Published online: 19 September 2020

\section{References}

1. Lee, H. J., Macbeth, A. H., Pagani, J. H. \& Young, W. S. III. Oxytocin: the great facilitator of life. Prog. Neurobiol. 88, 127-151 (2009).

2. Bartz, J. A., Zaki, J., Bolger, N. \& Ochsner, K. N. Social effects of oxytocin in humans: context and person matter. Trends Cogn. Sci. 15, 301-309 (2011).

3. Olff, M. et al. The role of oxytocin in social bonding, stress regulation and mental health: an update on the moderating effects of context and interindividual differences. Psychoneuroendocrinology 38, 1883-1894 (2013).

4. Shamay-Tsoory, S. G. \& Abu-Akel, A. The social salience hypothesis of oxytocin. Biol. Psychiatry 79, 194-202 (2016).

5. Shamay-Tsoory, S. G. et al. Intranasal administration of oxytocin increases envy and schadenfreude (gloating). Biol. Psychiatry 66, 864-870 (2009).

6. Rimmele, U., Hediger, K., Heinrichs, M. \& Klaver, P. Oxytocin makes a face in memory familiar. J. Neurosci. 29, 38-42 (2009).

7. Unkelbach, C., Guastella, A. J. \& Forgas, J. P. Oxytocin selectively facilitates recognition of positive sex and relationship words. Psychol. Sci. 19, 1092-1094 (2008).

8. Hu, J. et al. Oxytocin selectively facilitates learning with social feedback and increases activity and functional connectivity in emotional memory and reward processing regions. Hum. Brain Mapp. 36, 2132-2146 (2015).

9. Gamer, M., Zurowski, B. \& Buchel, C. Different amygdala subregions mediate valence-related and attentional effects of oxytocin in humans. Proc. Natl Acad. Sci. USA 107, 9400-9405 (2010).

10. Leppanen, J., Ng, K. W., Tchanturia, K. \& Treasure, J. Meta-analysis of the effects of intranasal oxytocin on interpretation and expression of emotions. Neurosci. Biobehav Rev. 78, 125-144 (2017).

11. Shahrestani, S., Kemp, A. H. \& Guastella, A. J. The impact of a single administration of intranasal oxytocin on the recognition of basic emotions in humans: a meta-analysis. Neuropsychopharmacology $\mathbf{3 8}$ 1929-1936 (2013). 
12. Savaskan, E., Ehrhardt, R., Schulz, A., Walter, M. \& Schachinger, H. Post-learning intranasal oxytocin modulates human memory for facial identity. Psychoneuroendocrinology 33, 368-374 (2008).

13. $\mathrm{Xu}, \mathrm{L}$. et al. Oxytocin enhances attentional bias for neutral and positive expression faces in individuals with higher autistic traits. Psychoneuroendocrinology 62, 352-358 (2015)

14. Blake, R. \& Logothetis, N. Visual competition. Nat. Rev. Neurosci. 3, 13-21 (2002).

15. Anderson, E. C. et al. Smiles may go unseen in generalized social anxiety disorder: evidence from binocular rivalry for reduced visual consciousness of positive facial expressions. J. Anxiety Disord. 27, 619-626 (2013).

16. Singer, N., Eapen, M., Grillon, C., Ungerleider, L. G. \& Hendler, T. Through the eyes of anxiety: dissecting threat bias via emotional-binocular rivalry. Emotion 12, 960-969 (2012).

17. Anderson, E., Siegel, E. H. \& Barrett, L. F. What you feel influences what you see: the role of affective feelings in resolving binocular rivalry. J. Exp. Soc. Psychol. 47, 856-860 (2011).

18. Yoon, K. L., Hong, S. W., Joormann, J. \& Kang, P. Perception of facial expressions of emotion during binocular rivalry. Emotion 9, 172-182 (2009).

19. Brascamp, J. W., Klink, P. C. \& Levelt, W. J. The 'laws' of binocular rivalry: 50 years of Levelt's propositions. Vis. Res. 109, 20-37 (2015).

20. Walle, K. M., Kyler, H. L., Nordvik, J. E., Becker, F. \& Laeng, B. Binocular rivalry after right-hemisphere stroke: effects of attention impairment on perceptual dominance patterns. Brain Cogn. 117, 84-96 (2017).

21. Anderson, E., Siegel, E. H., Bliss-Moreau, E. \& Barrett, L. F. The visual impact of gossip. Science 332, 1446-1448 (2011).

22. Blake, R. A neural theory of binocular rivalry. Psychol. Rev. 96, 145-167 (1989).

23. Dayan, P. A hierarchical model of binocular rivalry. Neural Comput. 10, 1119-1135 (1998).

24. Tong, F., Meng, M. \& Blake, R. Neural bases of binocular rivalry. Trends Cogn. Sci. 10, 502-511 (2006).

25. Said, C. P. \& Heeger, D. J. A model of binocular rivalry and cross-orientation suppression. PLoS Comput Biol. 9, e1002991 (2013)

26. van Loon, A. M. et al. GABA shapes the dynamics of bistable perception. Curr. Biol. 23, 823-827 (2013).

27. Seely, J. \& Chow, C. C. Role of mutual inhibition in binocular rivalry. J. Neurophysiol. 106, 2136-2150 (2011).

28. Freyberg, J., Robertson, C. E. \& Baron-Cohen, S. Reduced perceptual exclusivity during object and grating rivalry in autism. J. Vis. 15, 11 (2015).

29. Robertson, C. E., Kravitz, D. J., Freyberg, J., Baron-Cohen, S. \& Baker, C. I. Slower rate of binocular rivalry in autism. J. Neurosci. 33, 16983-16991 (2013).

30. Robertson, C. E., Ratai, E. M. \& Kanwisher, N. Reduced GABAergic action in the autistic brain. Curr. Biol. 26, 80-85 (2016).

31. Rubenstein, J. L. \& Merzenich, M. M. Model of autism: increased ratio of excitation/inhibition in key neural systems. Genes Brain Behav. 2, 255-267 (2003).

32. Nelson, S. B. \& Valakh, V. Excitatory/inhibitory balance and circuit homeostasis in autism spectrum disorders. Neuron 87, 684-698 (2015).

33. Leknes, S. et al. Oxytocin enhances pupil dilation and sensitivity to 'hidden' emotional expressions. Soc. Cogn. Affect Neurosci. 8, 741-749 (2013).

34. Duane, K. R. A brief history of stereoscopy. Wiley Interdiscip. Rev.: Computational Stat. 5, 334-340 (2013)

35. Auyeung, B. et al. Oxytocin increases eye contact during a real-time, naturalistic social interaction in males with and without autism. Transl. Psychiat. $\mathbf{5}$ e507 (2015)

36. Eckstein, M. et al. Oxytocin increases eye-gaze towards novel social and nonsocial stimuli. Soc. Neurosci. 14, 594-607 (2019).

37. Eikemo, M. et al. Sweet taste pleasantness is modulated by morphine and naltrexone. Psychopharmacol. (Berl.). 233, 3711-3723 (2016).

38. Loseth, G. E., Eikemo, M. \& Leknes, S. Effects of opioid receptor stimulation and blockade on touch pleasantness: a double-blind randomised trial. Soc. Cogn. Affect Neurosci. 14, 411-422 (2019).

39. Hubble, K. et al. Oxytocin reduces face processing time but leaves recognition accuracy and eye-gaze unaffected. J. Int Neuropsychol. Soc. 23, 23-33 (2017).

40. Domes, G., Kumbier, E., Heinrichs, M. \& Herpertz, S. C. Oxytocin promotes facial emotion recognition and amygdala reactivity in adults with Asperger syndrome. Neuropsychopharmacology 39, 698-706 (2014).

41. Hovey, D. et al. Emotion recognition associated with polymorphism in oxytocinergic pathway gene ARNT2. Soc. Cogn. Affect Neurosci. 13, 173-181 (2018).
42. Westberg, L. et al. Variation in the oxytocin receptor gene is associated with face recognition and its neural correlates. Front Behav. Neurosci. 10, 178 (2016).

43. Cattaneo, Z. et al. Congenital prosopagnosia is associated with a genetic variation in the oxytocin receptor (OXTR) gene: an exploratory study. Neuroscience 339, 162-173 (2016).

44. Skuse, D. H. et al. Common polymorphism in the oxytocin receptor gene (OXTR) is associated with human social recognition skills. Proc. Natl Acad. Sci. USA 111, 1987-1992 (2014).

45. Dieter, K. C., Brascamp, J., Tadin, D. \& Blake, R. Does visual attention drive the dynamics of bistable perception? Atten. Percept. Psychophys. 78, 1861-1873 (2016).

46. Domes, G. et al. Intranasal oxytocin increases covert attention to positive social cues. Psychol. Med. 43, 1747-1753 (2013).

47. Guastella, A. J., Mitchell, P. B. \& Dadds, M. R. Oxytocin increases gaze to the eye region of human faces. Biol. Psychiatry 63, 3-5 (2008).

48. Tollenaar, M. S., Chatzimanoli, M., van der Wee, N. J. \& Putman, P. Enhanced orienting of attention in response to emotional gaze cues after oxytocin administration in healthy young men. Psychoneuroendocrinology $\mathbf{3 8}$, 1797-1802 (2013)

49. Andari, E., Richard, N., Leboyer, M. \& Sirigu, A. Adaptive coding of the value of social cues with oxytocin, an fMRI study in autism spectrum disorder. Cortex 76, 79-88 (2016).

50. Rilling, J. K., Chen, X., Chen, X. \& Haroon, E. Intranasal oxytocin modulates neural functional connectivity during human social interaction. Am. J. Primatol. 80, e22740 (2018).

51. Nagamine, M., Yoshino, A., Miyazaki, M., Takahashi, Y. \& Nomura, S. Effects of selective 5-HT1A agonist tandospirone on the rate and rhythmicity of binocular rivalry. Psychopharmacol. (Berl.). 198, 279-286 (2008).

52. Carter, O. L. et al. Modulating the rate and rhythmicity of perceptual rivalry alternations with the mixed 5-HT2A and 5-HT1A agonist psilocybin. Neuropsychopharmacology 30, 1154-1162 (2005).

53. Yoshida, M. et al. Evidence that oxytocin exerts anxiolytic effects via oxytocin receptor expressed in serotonergic neurons in mice. J. Neurosci. 29, 2259-2271 (2009).

54. Dolen, G., Danvishzadeh, A., Huang, K. W. \& Malenka, R. C. Social reward requires coordinated activity of nucleus accumbens oxytocin and serotonin Nature 501, 179-184 (2013).

55. Tyzio, R. et al. Maternal oxytocin triggers a transient inhibitory switch in GABA signaling in the fetal brain during delivery. Science 314, 1788-1792 (2006).

56. Tyzio, R. et al. Oxytocin-mediated GABA inhibition during delivery attenuates autism pathogenesis in rodent offspring. Science 343, 675-679 (2014).

57. Leonzino, M. et al. The timing of the excitatory-to-inhibitory GABA switch is regulated by the oxytocin receptor via KCC2. Cell Rep. 15, 96-103 (2016).

58. Sala, M. et al. Pharmacologic rescue of impaired cognitive flexibility, social deficits, increased aggression, and seizure susceptibility in oxytocin receptor null mice: a neurobehavioral model of autism. Biol. Psychiatry 69, 875-882 (2011).

59. Ninan, I. Oxytocin suppresses basal glutamatergic transmission but facilitates activity-dependent synaptic potentiation in the medial prefrontal cortex. J. Neurochem. 119, 324-331 (2011).

60. Marlin, B. J., Mitre, M., D'Amour, J. A., Chao, M. V. \& Froemke, R. C. Oxytocin enables maternal behaviour by balancing cortical inhibition. Nature $\mathbf{5 2 0}$, 499-504 (2015).

61. Osako, Y., Otsuka, T., Taniguchi, M., Oka, T. \& Kaba, H. Oxytocin enhances presynaptic and postsynaptic glutamatergic transmission between rat olfactory bulb neurones in culture. Neurosci. Lett. 299, 65-68 (2001).

62. Harari-Dahan, O. \& Bernstein, A. A general approach-avoidance hypothesis of oxytocin: accounting for social and non-social effects of oxytocin. Neurosci. Biobehav Rev. 47, 506-519 (2014).

63. DeMayo, M. M., Song, Y. J. C., Hickie, I. B. \& Guastella, A. J. A review of the safety, efficacy and mechanisms of delivery of nasal oxytocin in children: therapeutic potential for autism and prader-willi syndrome, and recommendations for future research. Paediatr. Drugs 19, 391-410 (2017).

64. Bate, S. et al. Intranasal inhalation of oxytocin improves face processing in developmental prosopagnosia. Cortex 50, 55-63 (2014).

65. Servan, A., Brunelin, J. \& Poulet, E. The effects of oxytocin on social cognition in borderline personality disorder. Encephale 44, 46-51 (2018). 
66. Benner, S. \& Yamasue, H. Clinical potential of oxytocin in autism spectrum disorder: current issues and future perspectives. Behav. Pharmacol. 29, 1-12 (2018).

67. Leng, G. \& Ludwig, M. Intranasal oxytocin: myths and delusions. Biol. Psychiatry 79, 243-250 (2016).

68. Quintana, D. S. et al. Low-dose intranasal oxytocin delivered with breath powered device modulates pupil diameter and amygdala activity: a randomized controlled pupillometry and fMRI study. Neuropsychopharmacology $\mathbf{4 4}$ 306-313 (2019).
69. Spengler, F. B. et al. Kinetics and dose dependency of intranasal oxytocin effects on amygdala reactivity. Biol. Psychiatry 82, 885-894 (2017).

70. Quintana, D. S., Outhred, T., Westlye, L. T., Malhi, G. S. \& Andreassen, O. A. The impact of oxytocin administration on brain activity: a systematic review and meta-analysis protocol. Syst. Rev. 5, 205 (2016).

71. Carter, C. S. Oxytocin pathways and the evolution of human behavior. Annu Rev. Psychol. 65, 17-39 (2014). 